\title{
Acute respiratory failure and CNS-depressing drugs
}

\author{
J. M. B. GraY* \\ M.B. \\ D. A. HENRY* \\ M.B., M.R.C.P. \\ B. PAICE* \\ M.B., M.R.C.P. \\ G. GettinBy $\dagger$ \\ D.Phil. \\ F. MORAN* \\ M.B., M.S., F.R.C.P. \\ D. H. LAWSON* \\ M.D., F.R.C.P. \\ *Medical Division, Royal Infirmary, Glasgow, and $†$ Department of Mathematics, University of Strathclyde
}

\begin{abstract}
Summary
Data from a drug surveillance programme were analysed to estimate the frequency with which patients with a diagnosis of respiratory failure had been exposed to CNS-depressing drugs. Eleven out of 37 patients with respiratory failure had received such medication. A detailed comparison of these patients and controls admitted to hospital because of respiratory disease who did not develop respiratory failure failed to reveal significant differences in drug usage. This unexpected finding suggests that patients with respiratory disease of equal severity may vary greatly in their tendency to develop carbon dioxide retention following administration of drugs with respiratory depressant properties.
\end{abstract}

\section{Introduction}

Patients with recurrent respiratory problems, particularly chronic obstructive airways disease are at substantial risk of developing acute respiratory failure, a complication which carries with it a high morbidity and mortality.

It has been known for many years that drugs which depress central nervous system activity, such as narcotic analgesics, hypnotics and anxiolytic agents may precipitate the development of respiratory failure in such patients (Wilson, Hoseth and Dempsey, 1954). Nevertheless, this hazard seems to be insufficiently appreciated and these drugs continue to be used in patients with severe respiratory disease. In order to obtain an estimate of the frequency with which patients with respiratory failure have been exposed to such therapy, and to explore further the relationship between this condition and those drugs, the data accumulated during a drug surveillance programme in Glasgow Royal Infirmary, Medical Division, were reviewed.

\section{Methods}

During 1972-73, the drug treatment of all patients admitted to Glasgow Royal Infirmary, Medical Division, was documented. A record was kept of dose, route, frequency, starting and stopping dates of all drugs given to these patients. Demographic information including age, sex and hospital number were also recorded together with the duration of stay in hospital, outcome of admission (survival/ non-survival) and up to 4 discharge diagnoses per patient. The resulting information was then edited for completeness and entered on to magnetic tape for subsequent computer analyses.

All patients participating in the study were seen by J.M.B.G. and their case-notes reviewed on discharge. A diagnosis of respiratory failure was made only if the patient had a recorded arterial partial pressure of oxygen of less than $60 \mathrm{mmHg}$, and of carbon dioxide of greater than $50 \mathrm{mmHg}$.

A total of 5288 consecutive admissions were available for study. Of these, $587(11 \cdot 1 \%)$ had a diagnosis of respiratory disease (ICD nos 460-519 inclusive). The diagnoses most frequently made were chronic obstructive airways disease/chronic bronchitis (270 patients), bronchopneumonias (127 patients), acute bronchitis (73 patients) and asthma (72 patients).

The data from these patients were then reviewed and the proportion with a discharge diagnosis of acute respiratory failure determined. The hospital case-notes of all such patients were obtained and examined in detail. There were 37 patients with acute respiratory failure $(6 \cdot 3 \%$ of those with a discharge diagnosis of respiratory disease). The mean age of these patients was 62 years, $54 \%$ were male, their mean admission concentration of haemoglobin was $15.5 \mathrm{~g} / \mathrm{dl}$, of urea was $10 \mathrm{mmol} / \mathrm{l}$ and of creatinine 
was $93 \mu \mathrm{mol} / 1$. Arterial blood gas estimations showed their mean arterial $\mathrm{pH}$ to be $7 \cdot 35$, mean $\mathrm{Pa}, \mathrm{O}_{2} 43$ $\mathrm{mmHg}$ and $\mathrm{Pa}, \mathrm{CO}_{2} 61 \mathrm{mmHg}$. Five of these patients died while in hospital $(13 \%)$.

In order to explore in detail the relationship between acute respiratory failure and the administration of CNS-depressant drugs, each patient with respiratory failure was matched with 2 patients with a similar type of underlying respiratory disease who did not have respiratory failure (the controls). The criteria used for matching were age (within 5 years), survival while in hospital (yes/no), and nature of the underlying respiratory disease (emphysema, chronic bronchitis, asthma, etc.). The average age of the 74 control subjects was 61 years, $57 \%$ were male, admission $\mathrm{Hb}$ concentration was $14 \mathrm{~g} / \mathrm{dl}$, urea concentration was $8 \mathrm{mmol} / 1$ and creatinine concentration was $85 \mu \mathrm{mol} / \mathrm{l}$.

In order to compare the use of CNS-depressing drugs in those with respiratory failure and those without this diagnosis. drugs given in hospital to the cases before the diagnosis of respiratory failure were compared with those given to the controls during the same time interval. Thus, for example, if a case was in hospital $x$ days before the diagnosis of respiratory ailure was made, drugs given to this patient in the first $x$ days of the admission were compared with those given to each of the 2 controls for that case in the same time interval.

\section{Results}

Of the 37 patients with acute respiratory failure, $11(30 \%)$ received one or more drugs with CNSdepressant properties before the diagnosis of acute respiratory failure was made and, of the 74 control patients without acute respiratory failure, $20(27 \%)$ received such drugs in a comparable time interval. This difference could easily have arisen by chance.

In order to assess comparability between the cases and controls with respect to factors which are known to be related to severity of respiratory disease the data were submitted to a multivariate analysis. The factors included in this score were age, admission $\mathrm{Hb}$ concentration, admission urea concentration, use of antibiotics (none; ampicillin, tetracycline, cotrimoxazole; cephalosporin, aminoglycoside or other), use of digoxin (yes/no) and use of diuretics (yes/no). Low correlations were obtained between factors and even after these were taken into account, no differences were detectable between cases and controls. Similarly, multivariate scores produced for the cases and then the controls did not suggest that the consumption of drugs with CNS-depressing activity was a predicting factor of respiratory failure.

The drugs with CNS-depressant properties prescribed for the cases and controls are shown in Table 1, and a detailed description of the drugs implicated in the onset of acute respiratory failure is given in Table 2.

\section{Discussion}

It has been known for some time that CNS- $\overrightarrow{\vec{F}}$ depressing drugs may lead to respiratory failure in흐 patients with pre-existing severe respiratory disease. 믐

TABLE 1. CNS-depressing drugs prescribed for patients and $\frac{\rho}{\nabla}$ controls during comparable time interval

\begin{tabular}{lcc}
\hline \multicolumn{1}{c}{ Drug } & $\begin{array}{c}\text { Patients } \\
(n .37)\end{array}$ & $\begin{array}{c}\text { Controls } \\
(n .74)\end{array}$ \\
\hline Morphine & 3 & 0 \\
Dihydrocodeine & 2 & 7 \\
Benzodiazepines & 3 & 7 \\
Pentazocine & 0 & 3 \\
Others* & 3 & 3 \\
Any & $11(30 \%)$ & $20(27 \%)$ \\
\hline
\end{tabular}

*Chlormethiazole, phenobarbitone, premedications, chlordiazepoxide.

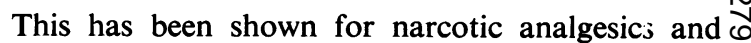
barbiturate hypnotics (Wilson et al., 1954; Foldes윽 Lumen and Tong, 1971) and more recently has been extended to include the benzodiazepine group of 3 drugs (Clark, Collins and Tong, 1971; Gaddie et al., 1972; Model and Berry, 1974). In 1971 it wass $\overrightarrow{0}$ reported from the Boston Collaborative Dris Surveillance Programme that drug-induced CN depression leading to respiratory failure was en ceeded only by hyperkalaemia and fluid overload ats a cause of drug-related deaths in medical patients in hospital (Shapiro et al., 1971).

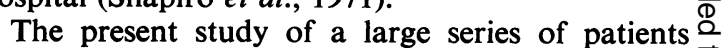
admitted to Glasgow Royal Infirmary Medical $\overrightarrow{0}$ Division, emphasizes that CNS-depressant drugs 3 continue to be given not infrequently to patients with respiratory disease. Indeed, $30 \%$ of those with acute respiratory failure had received one such drug 윽 in the time interval immediately preceding the diagnosis of respiratory failure. Notwithstanding 3 this observation, when a detailed comparison was made of the use of CNS-depressing drugs in those patients who developed acute respiratory failure and in a control group who did not, there were no $\frac{9}{5}$ significant differences in the use of these drugs $\rightarrow$ between the groups. This finding was unexpected. It is unlikely to be due to chance since there were $\tilde{N}$ substantial numbers of subjects in the comparison groups. Nor was it due to confounding by other $\mathrm{N}$ diseases present in the control group since both cases $\omega$ and controls were admitted as a result of their respiratory disease, and a review of subsidiary diagnosis did not reveal any unusual group of $\Phi$ secondary diagnoses in the control group. Since both $\stackrel{+}{+}$ the cases and controls were participating in a $\frac{0}{0}$ prospective study of drug use in medical in-patients, $\frac{\vec{\Phi}}{\mathbb{D}}$ there was no question of differential ascertainment of $\frac{?}{\mathbb{Q}}$ 
TABLE 2. CNS-depressing drugs implicated in onset of respiratory failure

$\begin{array}{ccc}\text { Patient } & \text { Drug(s) implicated } & \text { Comments } \\ \text { (age in years and sex) } & \end{array}$

1. $54 \mathrm{~F} \quad$ Dihydrocodeine bitartrate

2. $65 \mathrm{M}$

Morphine sulphate

3. $61 \mathrm{~F}$

Morphine sulphate

4. $74 \mathrm{~F} \quad$ Diamorphine

5. $56 \mathrm{M} \quad$ Papaveretum, Promazine

6. $58 \mathrm{M} \quad$ Chlormethiazole

7. $66 \mathrm{~F} \quad$ Nitrazepam

8. $72 \mathrm{~F} \quad$ Nitrazepam

9. $83 \mathrm{~F}$ Phenobarbitone

10. $62 \mathrm{~F}$ Dihydrocodeine
Chronic bronchitis, cor pulmonale. Exacerbation of bronchitis, received drug for 'back-ache' twice within $4 \mathrm{hr}$. Admitted unconscious and cyanosed. Required i.v. nikethamide. Recovered.

Chronic obstructive airways disease. Morphine given immediately before admission - reason unknown. Admitted deeply unconscious, cyanosed, constricted pupils. Nalorphine given. Recovered.

Rheumatic heart disease, chronic obstructive airways disease. Morphine given for 'distress' - became unconscious, cyanosed and developed respiratory failure. Nalorphine given with gradual improvement.

Congestive cardiac failure, chronic obstructive airways disease. Diamorphine given; reason unspecified. Became unconscious with respiratory depression. Nalorphine given with effect.

Chronic obstructive airways disease, cor pulmonale. Elective admission for respiratory investigation. Received premedication for pulmonary angiogram. Developed respiratory failure, tachycardia, respiratory arrest. Nalorphine given, spent 3 days on ventilator before recovery.

Chronic obstructive airways disease, congestive cardiac failure. Confused and obstreperous. Developed respiratory failure after $40 \mathrm{ml}$ chlormethiazole. Recovered gradually after drug withdrawal.

Chronic bronchitis, emphysema, status asthmaticus. After 2 days of nitrazepam $5 \mathrm{mg}$ at night developed drowsiness, orthopnoea, cyanosis and confusion. Arterial $\mathrm{PCO}_{2} 85 \mathrm{mmHg}$. Doxapram infusion required with marked improvement.

Chronic bronchitis in acute exacerbation with associated right sided cardiac failure: given nitrazepam $10 \mathrm{mg}$ nightly for 10 days. Gradually became confused and more cyanosed. Arterial $\mathrm{PCO}_{2} 80 \mathrm{mmHg}$. Responded upon stopping sedation.

Chronic bronchitis with congestive cardiac failure, receiving diuretics and antibiotics. Given phenobarbitone $30 \mathrm{mg}$ reason unspecified: developed respiratory failure possibly partly owing to effects of this drug.

Bronchopneumonia and chronic bronchitis given dihydrocodeine for pain in doses of $60 \mathrm{mg}, 5$ times in 4 days. On last day of therapy developed acute respiratory failure which responded to discontinuation of analgesic drug. drug use in hospital, although it is possible that this factor operated to a certain extent in ascertainment of pre-hospital drug use. Comparability of patients and controls with respect to severity of underlying respiratory disease at the time of admission is difficult to obtain. There is some evidence (in the form of higher presenting $\mathrm{Hb}$ and urea concentrations) that the cases were more severely ill than the controls on admission; nevertheless, even when this factor was taken into account using a multivariate analysis technique, no significant association between CNSdepressing drugs and respiratory depression was detected.

Although the number of patients exposed to morphine in this series was small, all patients so treated developed respiratory failure. Thus, it would seem likely that this group of drugs carries with it a substantial risk of producing severe respiratory failure in all recipients as has been reported previously.

The absence of a statistically significant association between respiratory failure and the use of the other CNS-depressant drugs such as the benzodiazepines, dihydrocodeine, and pentazocine could be accounted for by postulating that not all patients are at equal risk even given equal degrees of severity of respiratory disease. Such a conclusion receives some support from a small study of diazepam in lung 
disease (Kronenberg et al., 1975). Also the observation that there is a wide range of ventilatory responses to a given carbon dioxide stimulus both in healthy subjects (Irsigler, 1976) and in those with respiratory disease, is consistent with the proposition that patients might well vary considerably in respect of carbon dioxide retention following the administration of drugs with some respiratory-depressant properties.

\section{References}

Clark, T.J.H., Collins, J.V. \& Tong, D. (1971) Respiratory depression caused by nitrazepam in patients with respiratory failure. Lancet, ii, 737.

Foldes, F.F., Lumen, J.N. \& Benz, H.G. (1963) Prolonged respiratory depression caused by drug combinations. Journal of the American Medical Association, 183, 672.
Gaddie, J., Legge, J.S., Palmer, K.N.V., Petrie, J.C. \& Wood, R.A. (1972) Effect of nitrazepam in chronic obstructive bronchitis. British Medical Journal, 2, 688.

IRSIGLER, G.B. (1976) Carbon dioxide response lines in young adults: the limits of the normal response. American Review of Respiratory Disease, 114, 529.

Kronenberg, R.S., Cosio, M.G., Stevenson, J.E. \& Drage, C.W. (1975) The use of oral diazepam in patients with obstructive lung disease and hypercapnia. Annals of Internal Medicine, 83, 83.

Model, D.G. \& Berry, D.J. (1974) Effects of chlordiazepoxide in respiratory failure due to chronic bronchitis. os Lancet, ii, 869.

Shapiro, S., Slone, D., Lewis, G.P. \& Jick, H. Fatal drug reactions amongst medical in-patients. Journal of the $\vec{\omega}$ American Medical Association, 216, 467.

Wilson, R.H., Hoseth, W. \& Dempsey, M.E. (1954) Respir-Cृ atory acidosis. American Journal of Medicine, 17, 464. 